\title{
CIRCLE ACTIONS ON HOMOTOPY SPHERES NOT BOUNDING SPIN MANIFOLDS
}

\author{
BY \\ REINHARD SCHULTZ( $\left.{ }^{1}\right)$
}

\begin{abstract}
Smooth circle actions are constructed on odd-dimensional homotopy spheres that do not bound spin manifolds. Examples are given in every dimension for which exotic spheres of the described type exist.
\end{abstract}

A result of H. B. Lawson and S.-T. Yau [15] implies that homotopy spheres not bounding spin manifolds do not admit effective smooth $\mathrm{S}^{3}$ or $\mathrm{SO}_{3}$ actions. On the other hand, G. Bredon has shown that even-dimensional manifolds of this type can admit smooth circle actions [9]; in fact, such examples exist in every dimension of the form $8 k+2$, where $k>0$. Since homotopy spheres that are not spin boundaries only occur in dimensions $8 k+1$ and $8 k+2(k>0)$, it is natural to ask if smooth circle actions also exist on $(8 k+1)$-dimensional examples. We shall prove the answer is yes.

THEOREM. For every $k \geqslant 1$, there exists a homotopy $(8 k+1)$-sphere not bounding a spin manifold that admits an effective smooth $S^{1}$ action.

We shall prove this result by explicitly constructing semifree circle actions (see [10]) using surgery-theoretic methods. The motivation for such an approach is that it yields an extremely simple proof of Bredon's result in the even-dimensional case (see Remark 3.11 below). Several technical facts make the odd-dimensional case more complicated; the most important are (i) the indecomposability of the $(8 k+1)$-dimensional framed bordism classes of the homotopy spheres considered, and (ii) the need to calculate surgery obstructions for normal maps into certain simply connected $8 k$-manifolds. Needless to say, the bulk of this paper is devoted to circumventing the first problem and studying the second. These are done using the machinery of Adams' $J(X)$ papers (e.g., [2] , [3]) and the validity of the Adams conjecture [19].

REMARKS. 1. It is natural to ask whether tori of rank $\geqslant 2$ can act smoothly on the manifolds considered; I do not know the answer to this question.

2. The exotic spheres considered here provide examples of odd-dimensional spin manifolds with smooth $S^{1}$ actions but no Riemannian metric having positive scalar curvature (compare [15]).

Received by the editors October 7, 1974.

AMS (MOS) subject classifications (1970). Primary 57D65, 57E25; Secondary 57D20, 57D55, 55 F50.

( ${ }^{1}$ )The author was partially supported by NSF Grant GP-19530A2. 
1. Decomposability of $p_{*} \mu_{k}$. We begin by recalling from [5] that the image of $\Omega_{8 k+\epsilon}^{\mathrm{fr}}$ in $\Omega_{8 k+\epsilon}^{\mathrm{Spin}}(\epsilon=1$ or 2$)$ is cyclic of order 2. Furthermore, the odddimensional spin classes have preimages $\mu_{k} \in \Omega_{8 k+1}^{\mathrm{fr}}$ of order two defined in [3], and the even-dimensional classes have preimages $\mu_{k} \eta$ (where $0 \neq \eta \in \Omega_{1}^{\mathrm{fr}} \cong Z_{2}$ ) [3], [5]. Since $\mu_{k}^{*} \widetilde{K O}^{*}\left(S^{0}\right) \rightarrow \widetilde{K O}^{*}\left(S^{8 k+1}\right)$ is nontrivial [3], it follows from the results of [3] that $\mu_{k}$ is indecomposable in $\Omega_{*}^{\mathrm{fr}}$. On the other hand, for some time it has been known that the image of $\mu_{k}$ in $\Omega_{*}^{\text {Spin }}$ is decomposable (Milnor treats the cases $k=1,2$ in [18] and the rest follow from [3] and the conjecture in $\left[18\right.$, p. 61]). For our purposes it is necessary to know that $p_{*} \mu_{k}$ is decomposable as $M_{k} \eta$ in $\pi_{*}(F / O)$. Although this is undoubtedly known to many people, no proof appears to be in the literature, and therefore we shall record one in this section.

D. Sullivan has pointed out that the Adams conjecture yields splittings of the the spaces $F$ and $F / O$ at every prime $p$; namely,

$$
\begin{gathered}
F_{(p)} \cong J_{(p)} \times \operatorname{Cok} J_{(p)}, \\
F / O_{(p)} \cong B S O_{(p)} \times \operatorname{Cok} J_{(p)}
\end{gathered}
$$

(the first splitting for $p$ odd was mentioned in [24], and the general cases are treated in [17]).

These splittings are related by the fact that the composite $\operatorname{CokJ}(p) \rightarrow F_{(p)}$ $\rightarrow F / O_{(p)} \rightarrow \operatorname{CokJ}(p)$ is (homotopically) the identity. Furthermore, the inclusions of $J_{(p)}$ and $B S O_{(p)}$ fit into a long commutative diagram,

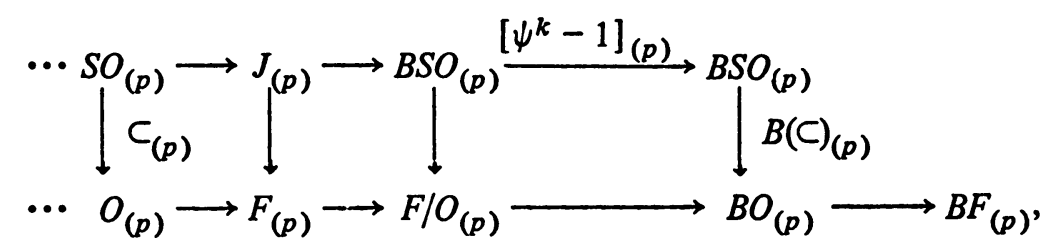

where the horizontal rows are extended fibration sequences, $\psi^{k}$ is an Adams operation, and $k$ is suitably chosen with respect to $p$ (see [23] for $p$ odd; if $p=2$, $k=3$ is the customary value [14], [17]).

Recall that if $p$ is odd then $\pi_{*}\left(J_{(p)}\right)$ is equal to the classical image of the $p$-primary $J$-homomorphism [23], while if $p=2$ then $\pi_{*}\left(J_{(p)}\right)$ contains the image of the 2-primary $J$-homomorphism together with $Z_{2}$-summands in dimensions $8 k+1,8 k+2(k \geqslant 1)$ corresponding to $\mu_{k}$ and $\mu_{k} \eta$ (compare [14]). Since $p_{*} \mu_{k}$ and $p_{*} \mu_{k} \eta$ are nonzero in $\pi_{*}(F / O)$, the following observation is immediate:

(1.3) With respect to the splitting of $F / O_{(2)}$ given in (1.2), the first coordinates of the images of $p_{*} \mu_{k}$ and $p_{*} \mu_{k} \eta$ in $\pi_{*}\left(F / O_{(2)}\right)$ are nonzero and the second coordinates are zero.

From the above considerations the desired factorization of $P_{*} \mu_{k}$ follows readily: 

satisfying

Proposition 1.4. Suppose $k \geqslant 1$. Then there is a class $M_{k} \in \pi_{8 k}(F / O)$

(i) $M_{k}$ passes to a generator of $\pi_{8 k}(F / O) /$ Torsion $\cong Z$.

(ii) $M_{k} \eta=p_{*} \mu_{k}$.

ProOF. Let $M_{k}^{\prime} \in \pi_{8 k}(F / O)_{(2)}$ correspond to a generator of $\pi_{8 k}(B S O)_{(2)}$ under splitting (1.2). Then $M_{k}^{\prime} \eta=$ image of $p_{*} \mu_{k}$ by (1.3) and the nontriviality of $\eta^{*}: \pi_{8 k}(B S O) \rightarrow \pi_{8 k+1}(B S O)$ [8]. Let $M_{k}^{\prime \prime} \in \pi_{8 k}(F / O)$ be chosen so that image $M_{k}^{\prime \prime}=a M_{k}^{\prime}$ for some odd integer $a$; since $M_{k}^{\prime} \eta=$ image $p_{*} \mu_{k}$, it follows that $M_{k}^{\prime \prime} \eta-p_{*} \mu_{k}$ is odd torsion. But $p_{*} \mu_{k}$ has order 2 by construction, and $M_{k}^{\prime \prime} \eta$ also has order 2 because $\eta$ does and the composition $\pi_{p+q}\left(S^{p}\right) \times \pi_{p}(F / O)$ $\rightarrow \pi_{p+q}(F / O)$ is bilinear (compare [20]); thus the above difference has order two and accordingly is zero.

Consider the class of $M_{k}^{\prime \prime}$ in $\pi_{8 k}(F / O) /$ Torsion. Since $p_{*} \mu_{k}$ is not divisible by 2 and the homotopy composition is bilinear, $\left[M_{k}^{\prime \prime}\right]$ is not divisible by 2 . Thus if $M_{k}^{\prime \prime \prime} \in \pi_{8 k}(F / O)$ represents a generator and $\left[M_{k}^{\prime \prime}\right]$ is divisible by the odd integer $b$, then $M_{k}^{\prime \prime}-b M_{k}^{\prime \prime \prime}$ is torsion in $\pi_{8 k}(F / O)$. Finally, the element $M_{k}=M_{k}^{\prime \prime}+$ $(1-b) M_{k}^{\prime \prime \prime}$ has all the desired properties (notice that $b \zeta \eta=\zeta \eta$ since $2 \eta=0$ ).

2. Pontrjagin class calculations. In order to prove our main result, it is necessary to show that certain types of normal maps into certain 1-connected $8 k$ manifolds (e.g., $S^{2} \times C P^{3}$ ) are normally bordant to homotopy equivalences. Since the obstruction to finding such bordisms is an index difference [11], it is obviously necessary to study the rational Pontrjagin classes of fiber homotopically trivial bundles over the relevant $8 k$-manifolds. The necessary facts are collected in this section; everything done here is a straightforward application of basic results on $K$-theory found in [1]-[4], [7] and the Adams conjecture.

Let $k \geqslant 1$ be an integer, and let $s$ be another integer satisfying $1 \leqslant s \leqslant 2 k-$ 1 ; we wish to find the Pontrjagin classes of certain fiber homotopically trivial vector bundles over the $8 k$-dimensional complex

$$
X_{k, s}=S^{8 k-4 s-2}\left(C P^{2 s+1} / C P^{2 s-2}\right) \text {. }
$$

It is well known (compare [13], [22]) that this cell complex is homotopically the mapping cone of a function

$$
f+\eta: S^{8 k-1} \vee S^{8 k-3} \rightarrow S^{8 k-4},
$$

where $\eta$ is the suspended Hopf map (as usual) and $f$ is partially determined as follows:

Proposition 2.1. The complex e-invariant of $f: S^{8 k-1} \rightarrow S^{8 k-4}$ is given by $e_{\mathrm{C}}(f)=(s+1) / 12$.

Proof. By the results of $[3, \S 7]$, it suffices to calculate the Chern character of an element in $\widetilde{K}(C(f))$ that restricts to a generator of $\widetilde{K}\left(S^{8 k-4}\right)=Z(C(f)=$ 
mapping cone of $f$ ). On the other hand, by the above description of $X_{k, s}$ it is clear that there is a map $C(f) \rightarrow X_{k, s}$ inducing a surjection of integral singular cohomology, and thus it suffices to calculate Chern characters for elements of $\widetilde{K}\left(X_{k, s}\right)$. But this is a simple application of the results in [7] and the multiplicativity of the Chern character.

By the above proposition and the remarks of $[3$, p. 41$]$, there is a complex vector bundle $\zeta$ on $C(f)$ such that

$$
\operatorname{ch} \zeta=h_{8 k-4}+(s+1) h_{8 k} / 12
$$

( $h_{m}$ is defined as in [3, p. 41]). Since $C(f)$ is a suspension by standard homotopytheoretic results, the decomposable Chern classes vanish and we have

$$
\operatorname{ch} \zeta=c_{4 k-2}(\zeta) /(4 k-3) !+c_{4 k}(\zeta) /(4 k-1) ! .
$$

Combining (2.2) and (2.3), we obtain the characteristic classes of $\zeta$ :

$$
\begin{gathered}
c_{4 k-2}(\zeta)=(4 k-3) ! h_{8 k-4}, \quad c_{4 k}(\zeta)=\frac{(s+1)(4 k-1) !}{12} h_{8 k} \\
p_{2 k-1}(\mathrm{r} \zeta)=-2(4 k-3) ! h_{8 k-4}, \quad p_{2 k}(\mathrm{r} \zeta)=\frac{(s+1)(4 k-1) !}{6} h_{8 k}
\end{gathered}
$$

(r's denotes the underlying real vector bundle.)

Actually, we are not primarily interested in $\mathbf{r} \zeta$, but rather in a fiber homotopically trivial vector bundle whose restriction to $\widetilde{K O}\left(S^{8 k-4}\right)=Z$ generates the kernel of the $J$-homomorphism, at least when localized at 2 . According to the Adams conjecture, such a vector bundle is given by

$$
3^{e}\left(\psi_{R}^{3} \mathrm{r} \zeta-\mathrm{r} \zeta\right)
$$

for some sufficiently large integer $e$.

(Note. For this purpose it suffices to use the weak Adams conjecture proved in [2] together with the identity $\psi_{R}^{3} \mathrm{r} \zeta=\mathrm{r} \psi_{C}^{3} \zeta$ [4].)

If $\sigma \in \widetilde{K}(C(f))$ is the pullback of the generator of $\widetilde{K}\left(S^{8 k}\right)=Z$, a result of Adams [3, p. 41] and Proposition 2.1 imply that

$$
\psi_{R}^{3} \mathrm{r} \xi-\mathrm{r} \zeta=\left(3^{4 k-2}-1\right) \mathrm{r} \zeta+3^{4 k-3} \cdot 2 \cdot(s+1) \mathrm{r} \sigma ;
$$

since $p_{2 k}(\mathrm{r} \sigma)=2(4 k-1) ! h_{8 k}$, the Pontrjagin classes of $(2.6)$ are easily obtainable from (2.5) and (2.7) (alternatively, one can use Adams' formula for ch $\psi^{k} \zeta[1$, Theorem 5.1 (vi)] directly). Finally, we record the following simple fact.

Proposition 2.8. The "restriction" map from $K O\left(X_{k, s}\right)$ to $K O(C(f))$ is an isomorphism.

This follows immediately from the triviality of $\pi_{i}(B O)$ for $i \equiv 5,6,7 \bmod 8$.

3. Proof of main result. Let $\alpha=\psi_{R}^{3} \mathrm{r} \zeta-\mathrm{r} \zeta$, and let $\beta=\left(3^{4 k}-1\right) \mathrm{r} \sigma / 2=$ $\psi_{R}^{3} \sigma^{\prime}-\sigma^{\prime}$, where $\sigma^{\prime} \in \widetilde{K O}(C(f))$ is the pullback of a generator of $\widetilde{K O}\left(S^{8 k}\right)=Z$ 
such that $\sigma^{\prime} \otimes C=\sigma$ (and hence $2 \sigma^{\prime}=\mathrm{r} \sigma$ ); $\sigma^{\prime}$ exists since $\pi_{8 k-1}(U / O)=0$ by Bott periodicity [8]. Using the isomorphism of Proposition 2.8, these vector bundles may be pulled back to vector bundles on $S^{8 k-4 s-2} \times C P^{2 s+1}\left(=M_{k, s}\right.$ henceforth), and we shall also call these pullbacks $\alpha$ and $\beta$. By the Adams conjecture, $3^{e} \alpha$ and $3^{e} \beta$ are fiber homotopically trivial for suitably large $e$, and thus for each pair of integers $(x, y)$ there is a normal map

$$
f: X^{8 k} \rightarrow M_{k, s}
$$

for which $\tau_{X} \oplus f^{*} v_{M} \cong f^{*}\left(3^{e} x \alpha \oplus 3^{e} y \beta\right)$. Since the index of the 1-connected manifold $M_{k, s}$ is zero, 8 times the surgery obstruction of $f$ is merely

$$
\begin{aligned}
\operatorname{index}(X) & =\left\langle L_{2 k}\left(\tau_{X}\right),[X]\right\rangle=\left\langle L_{2 k}\left(f^{*} \tau_{M} \oplus f^{*}\left(3^{e} x \alpha \oplus 3^{e} y \beta\right)\right),[X]\right\rangle \\
& =\left\langle L_{2 k}\left(\tau_{M} \oplus 3^{e} x \alpha \oplus 3^{e} y \beta\right),[M]\right\rangle .
\end{aligned}
$$

The multiplicative property of the $L$ classes and the triviality of $p_{j}(\alpha)$ and $p_{j}(\beta)$ for $j<2 k-1$ then yield

LEMMA 3.1. The surgery obstruction of $f_{(x, y)}$ vanishes if and only if

$$
L_{2 k}\left(3^{e} x \alpha \oplus 3^{e} y \beta\right)+L_{1}\left(C P^{2 s+1}\right) L_{2 k-1}\left(3^{e} x \alpha \oplus 3^{e} y \beta\right)=0 .
$$

Since $\alpha$ and $\beta$ are induced from bundles over suspensions, the above expression is additive in $3^{e}, x$, and $y$. In particular, (3.2) is equivalent to the analogous equation with all coefficients of the form $3^{e}$ suppressed. Furthermore, for $\gamma$ $=x \alpha \oplus y \beta$ we then have $L_{j}(\gamma)=l_{j} p_{j}(\gamma)$ (since the decomposable terms drop out), where

$$
l_{m}=\frac{2^{2 m+1}\left(2^{2 m-1}-1\right)}{(2 m-1) ! j_{4 m}} \operatorname{num}\left(B_{m} / 4 m\right)
$$

(compare [12, p. 292]), with $j_{4 m}=\operatorname{order} \widetilde{J}\left(S^{4 m-1}\right)$. Therefore, if we use the calculations of $\S 2$, together with

$$
L_{1}\left(C P^{2 s+1}\right)=(2 s+2) t^{2} / 3
$$

(where $t \in H^{2}\left(C P^{2 s+1}\right)$ is a generator) and $L_{2 k-1}(\beta)=0$, we may rewrite the vanishing condition of (3.2) in the following numerical form.

$$
\begin{gathered}
l_{2 k}\left(\left[y \cdot(4 k-1) ! \cdot\left(3^{4 k}-1\right)\right]+\left[x \cdot 3^{4 k-3} \cdot 4 \cdot(s+1) \cdot(4 k-1) !\right]\right. \\
\left.+\left[\frac{x \cdot\left(3^{4 k-2}-1\right) \cdot(s+1) \cdot(4 k-1) !}{6}\right]\right) \\
=\frac{l_{2 k-1} \cdot x \cdot 2 \cdot(4 k-3) ! \cdot\left(3^{4 k-2}-1\right) \cdot(2 s+2)}{3} .
\end{gathered}
$$

If we substitute for $l_{j}$ using (3.3), divide both sides of the equation by $2^{4 k+1}$, and rearrange the terms, we obtain 


$$
\begin{aligned}
&= x\left\{\frac{\left(3^{4 k-2}-1\right) \cdot\left(2^{4 k-3}-1\right) \cdot \operatorname{num}\left(B_{2 k-1} / 8 k-4\right)}{j_{8 k-4} \cdot 3}\right. \\
&\left.-\frac{\left(2^{4 k-1}-1\right) \cdot \operatorname{num}\left(B_{2 k} / 8 k\right) \cdot\left(3^{4 k}-1\right)}{j_{8 k} \cdot 6}\right\} \cdot(s+1) .
\end{aligned}
$$

The above equation has the form $y a=x b$, where $a$ and $b$ are rational numbers; we wish to calculate the 2-adic valuations of these coefficients, which are given by the formula

$$
c=2^{-\left(\log _{2} \nu_{2}(c)\right)} p / q,
$$

where $p$ and $q$ are odd integers.

Since $v_{2}\left(j_{4 m}\right)=v_{2}\left(3^{2 m}-1\right)$ (compare [3]) and the numerator of $B_{m} / 4 m$ is odd (the denominator is divisible by 8 ), we have $v_{2}(a)=1$. To calculate $v_{2}(b)$, write $b=\left(b^{\prime}-b^{\prime \prime}\right)(s+1)$, where $b^{\prime}$ and $b^{\prime \prime}$ are the two positive rational coefficients in the expression enclosed within braces on the right-hand side of (3.5). By the same reasoning used for $\nu_{2}(a)$, we see that

$$
\nu_{2}\left(b^{\prime}\right)=1, \quad \nu_{2}\left(b^{\prime \prime}\right)=2 .
$$

It follows that $v_{2}(b)=2 v_{2}(s+1)$. Therefore $v_{2}(a) \leqslant v_{2}(b)$ reduces to the inequality

$$
s \not=3 \bmod 4 \text {. }
$$

FACT (3.8). If (3.7) holds, then there is an integral solution of the (equivalent) equations (3.2), (3.4), or (3.5) with $y$ odd. For then $\nu_{2}(a) \leqslant v_{2}(b) \mathrm{im}$ plies

$$
a=2^{\alpha}(p / q), \quad b=2^{\beta}(r / t)
$$

where $p, q, r, t$ are odd integers and $\alpha \geqslant \beta$, so that $(x, y)=\left(2^{\alpha-\beta} p t, q r\right)$ solves the equation (s).

We can now prove a strong form of the main result.

THEOREM 3.9. Let $k \geqslant 1$ and $q \leqslant 2 k$; assume that $q \neq 1$ and $q \neq 0 \bmod 4$. Then there is a semifree circle action on some exotic $(8 k+1)$-sphere not bounding a spin manifold whose fixed point set is an ordinary sphere of codimension $4 q$.

REMARK. By a result in [21], no such actions exist for codimensions of the form $4 q+2$ ( $q^{4}$ arbitrary). Levine has shown that codimension 4 is not realizable either ([16]; see [22] for another proof).

Proof. Let $s=q-1$; then $s \geqslant 1$ since $q \geqslant 2$. We claim the normal maps $f_{(x, y)}$ may be chosen so that $f_{(x, y)} \mid f_{(x, y)}^{-1}\left(D_{-}^{8 k-4 s-2} \times C P^{2 s+1}\right)$ is a diffeomorphism. To show this, it suffices to show that the "classifying bundle" of such a 
normal map (see [11] for example), which is an element of [ $S^{8 k-4 s-2} \times$ $\left.C P^{2 s+1}, F / O\right]$, may be chosen to lie in the image of

$$
\begin{aligned}
& {\left[S^{8 k-4 s-2} \times C P^{2 s+1} / D_{-}^{8 k-4 s-2} \times C P^{2 s+1}, F / O\right]} \\
& \cong\left[D^{8 k-4 s-2} \times C P^{2 s+1} / S^{8 k-4 s-3} \times C P^{2 s+1}, F / O\right] \quad \text { (by excision) } \\
& \cong\left[S^{8 k-4 s-2} \wedge\left(C P^{2 s+1} \vee S^{0}\right), F / O\right] \quad \text { (by a canonical homeomorphism [6]) }
\end{aligned}
$$

(see [10] or [25, pp. 106-107] for background information). But, by construction, the bundles $3^{e} x \alpha+3^{e} y \beta$ are induced from fiber homotopically trivial vector bundles over $S^{8 k-4 s-2}\left(C P^{2 s+1} / C P^{2 s-2}\right)$, and hence canonical choices of the desired type are always possible.

If we excise the open submanifolds over which $f_{(x, y)}$ is a diffeomorphism by construction, we obtain a canonically associated relative normal map of pairs

$$
f^{\prime}:\left(X_{0}, \partial X_{0}\right) \rightarrow\left(D^{8 k-4 s-2}, s^{8 k-4 s-3}\right) \times C P^{2 s+1}
$$

that is a diffeomorphism on the boundary. The relative surgery obstruction for this relative normal map $f^{\prime}$ agrees with the (absolute) surgery obstruction for $f$ since $D^{8 k-4 s-2} \times C P^{2 s+1}$ and $S^{8 k-4 s-2} \times C P^{2 s+1}$ are both 1-connected (compare [11] or [25, pp. 106-107]), and thus $f_{(x, y)}^{\prime}$ is normally bordant to a homotopy equivalence if and only if $(x, y)$ satisfies equations (3.2), (3.4), (3.5). By (3.8), the comments after (3.7), and the immediately preceding remarks, we may choose $(x, y)$ so that $y$ is odd, and the surgery obstruction for $f_{(x, y)}^{\prime}$ vanishes.

For the above choice of $(x, y)$, assume the normal map $f_{(x, y)}^{\prime}$ is chosen within the appropriate cobordism class to be a homotopy equivalence (there is an essentially unique choice up to $h$-cobordism by the surgery exact sequence [11], [25] since $L_{8 k+1}(1)=0$ ); using this representative, a semifree circle action may be constructed on some homotopy $(8 k+1)$-sphere by the following standard process (compare [10] or [22]): Take the pullback of the canonical principal $S^{1}$ bundle

$$
D^{8 k-4 s-2} \times S^{2 s+3} \rightarrow D^{8 k-4 s-2} \times C P^{2 s+1}
$$

with respect to $f^{\prime}$. This yields a homotopy equivalence of total spaces

$$
f^{\prime \prime}:(Y, \partial Y) \rightarrow\left(D^{8 k-4 s-2}, s^{8 k-4 s-3}\right) \times S^{4 s+3}
$$

that is a diffeomorphism on the boundary and is $S^{1}$-equivariant. The manifold

$$
\Sigma_{(x, y)}^{8 k+1}=Y U_{f^{\prime \prime} \mid \partial y} S^{8 k-4 s-3} \times D^{4 s+4}
$$

(trivial action on the first coordinate of $S \times D$, complex multiplication on the second) formed by equivariant identification is then a smooth $S^{1}$-manifold, and it admits a smooth semifree action with $S^{8 k-4 s-3}=S^{8 k+1-4 q}$ as the fixed 
point set. By van Kampen's theorem and a Mayer-Vietoris argument, $\Sigma$ is a homotopy sphere.

It remains to show that $\Sigma^{8 k+1}$ does not bound a spin manifold. But the Pontrjagin-Thom invariant of $\Sigma$ is recoverable from the classifying bundle of $f^{\prime}$ as follows. The natural map

$$
\begin{aligned}
\pi^{*}:\left[S^{8 k-4 s-2}\right. & \left.\wedge\left(C P^{2 s+1} \vee S^{0}\right), F / O\right] \\
& \rightarrow\left[S^{8 k-4 s-2} \wedge\left(S^{4 s+3} \vee S^{0}\right), F / O\right] \\
& \cong \pi_{8 k+1}(F / O) \times \pi_{8 k-4 s-2}(F / O)
\end{aligned}
$$

induced by $\pi: S^{4 s+3} \rightarrow C P^{2 s+1}$ sends the classifying bundle of $f^{\prime}$ to that of $f^{\prime \prime}$ (compare [25, pp. 186, 187, 242]), and the elementary attaching considerations imply that the first coordinate of the classifying bundle for $f^{\prime \prime}$ is the PontrjaginThom invariant up to appropriate sign conventions forced by choices of orientation.

Explicit choices for the normal maps $f_{(x, y)}^{\prime}$ are obtainable via the Adams conjecture; for the reasoning used to obtain the splittings in $\S 1$ in fact induces a commutative diagram (modulo maps of infinite filtration)

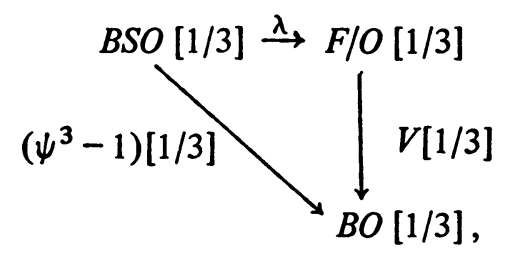

where $\cdots[1 / 3]$ denotes localization away from $3, V$ classifies the universal stable bundle over $F / O$, and all maps are $H$-maps (note that $[X, H[1 / 3]] \cong[X, H] \otimes$ $Z[1 / 3]$ for $X$ finite and the above $H)$. In the notation of $\S 2$, let $\alpha^{\prime}$ and $\beta^{\prime}$ denote the images of $\zeta$ and $\eta$ under the canonical maps

$$
\left[X_{k, s}, B S O\right] \rightarrow\left[X_{k, s}, B S O\right] \otimes Z[1 / 3] \stackrel{\lambda^{*}}{\rightarrow}\left[X_{k, s} F / O\right] \otimes Z[1 / 3],
$$

and choose $e$ so large that $3^{e} \alpha^{\prime}, 3^{e} \beta^{\prime} \in$ Image $\left\{\left[X_{k, s} F / O\right] \longrightarrow\left[X_{k, s,} F / O\right] \otimes Z[1 / 3]\right\}$. Then $3^{e}\left(x \alpha^{\prime}+y \beta^{\prime}\right)$ is a convenient explicit choice for $f_{(x, y)}^{\prime}$.

To determine the Pontrjagin-Thom invariant of $\Sigma_{(x, y)}^{8 k+1}$ for the above choice of (surgically unobstructed) normal map, by naturality it suffices to determine the image of $3^{e}\left(x \zeta+y \sigma^{\prime}\right)$ in $\left(\pi_{8 k+1}(B S O) \otimes Z[1 / 3]\right) \times\left(\pi_{8 k-4 s-2}(B S O) \otimes Z[1 / 3]\right)$ under the map $\pi^{*}$ analogous to (3.10) for $B S O[1 / 3]$. By Bott periodicity the first factor is $Z_{2}$ and the second is $Z_{2}$ or 0 , and by (1.3) and Proposition 1.4 it suffices to show that the first coordinate of $\pi^{*}\left(3^{e}\left(x \zeta+y \sigma^{\prime}\right)\right)$ is nonzero. Since the product group has exponent two, the coefficient $3^{e}$ acts as the identity and can be suppressed; thus we need only consider $x \pi^{*} \zeta+y \pi^{*} \sigma^{\prime}$. First, we claim that $\pi^{*} \zeta=0$; this is true because $\zeta$ has a complex structure, the restriction maps $\widetilde{K}\left(S^{m} C P^{2 s+2}\right) \rightarrow \widetilde{K}\left(S^{m} C P^{2 s+1}\right)$ are onto [4] , and $C P^{2 s+2}$ is the mapping cone 
of $\pi$. On the other hand, $\sigma^{\prime}$ is the pullback of the generator of $\pi_{8 k}(B S O)$ under the .collapsing map

$$
S^{8 k-4 s-2}\left(C P^{2 s+1} / C P^{2 s-2}\right) \rightarrow S^{8 k}
$$

and $\pi^{*} \sigma^{\prime}$ is thus the composite of this generator with the map

$$
h: S^{8 k+1} \rightarrow S^{8 k-4 s-2}\left(C P^{2 s+1}\right) \rightarrow S^{8 k}
$$

localized away from 3. It is well known that $h$ is the suspended Hopf map (compare [13] or [22]), so that $\sigma^{\prime} h$ is the nonzero element of $\pi_{8 k+1}(B S O)=Z_{2}$ (see [8] ); since tensoring with $Z[1 / 3]$ is an isomorphism for finite 2-primary groups, the first coordinate of $\pi^{*} \sigma^{\prime}$ is also nonzero. Since $y$ is odd, these calculations, Proposition 1.4 and (1.3) conclude the proof of Theorem 3.9.

Complement 3.11. A similar but simpler argument proves the analogous result for exotic $(8 k+2)$-spheres not bounding spin manifolds with no restrictions on $q$ except the obvious one, $4 q \leqslant 8 k+2$. Explicitly, take the normal map whose classifying bundle $\mu_{k}^{\#}$ in $\left[S^{8 k-4 s+1}\left(C P^{2 s+1} \vee S^{0}\right), F / O\right]$ is the pullback of the element $p_{*} \mu_{k} \in \pi_{8 k+1}(F / O)$. Since odd-dimensional 1-connected surgery groups vanish, the relative normal map corresponds to a homotopy equivalence that is a diffeomorphism on the boundary. As in the preceding paragraph, the first coordinate of $\pi^{*} \mu_{k}^{\#}$ is $p_{*} \mu_{k} \eta$, and thus the previously described construction for semifree circle actions yields the desired examples. This method of construction is basically equivalent to Bredon's [9]. Of course, the important simplifying difference is that odd-dimensional simply connected surgery is always possible.

\section{REFERENCES}

1. J. F. Adams, Vector fields on spheres, Ann. of Math. (2) 75 (1962), 603-632. MR 25 \#2614.

2. - On the groups $J(X)$. I, Topology 2 (1963), 181-195. MR 28 \#2553.

3. - On the groups $J(X)$. IV, Topology 5 (1966), 21-71; correction, ibid. 7 (1968), 331. MR 33 \#6628; 37 \#5874.

4. J. F. Adams and G. Walker, On complex-Stiefel manifolds, Proc. Cambridge Philos. Soc. 61 (1965), 81-103. MR 30 \#1516.

5. D. W. Anderson, E. H. Brown and F. P. Peterson, The structure of the Spin cobordism ring, Ann. of Math. (2) 86 (1967), 271-298. MR 36 \#2160.

6. M. F. Atiyah, Thom complexes, Proc. London Math. Soc. (3) 11 (1961), 291-310. MR. 24 \#A1727.

7. - K-theory, Benjamin, New York, 1967. MR 36 \#7130.

8. R. Bott, The stable homotopy of the classical groups, Ann. of Math. (2) 70 (1959), 313-337. MR 22 \#987.

9. G. Bredon, $A \Pi_{*}-$ module structure for $\Theta_{*}$ and applications to transformation groups, Ann. of Math. (2) 86 (1967), 434-448.

10. W. Browder, Surgery and the theory of differentiable transformation groups, Proc. Conf. on Transformation Groups (New Orleans, La., 1967), Springer, New York, 1968, pp. 1-46. MR 41 \#6242.

11. - Surgery on simply connected manifolds, Ergebnisse der Mathematik und ihrer Grenzgebiete, Band 65, Springer-Verlag, New York, 1972.

12. G. Brumfiel, On the homotopy groups of $B P L$ and $P L / O$, Ann. of Math. (2) 88 (1968), 291-311. MR 38 \#2775. 
13. G. Brumfiel, Differentiable $s^{1}$ actions on homotopy spheres, Univ. of Calif., Berkeley, 1968 (mimeographed).

14. R. R. Clough, The $Z_{2}$-cohomology of a candidate for $B_{\operatorname{Im}(J)}$, llinois J. Math. 14 (1970), 424-433. MR 41 \#7674.

15. H. B. Lawson and S.-T. Yau, Scalar curvature, nonabelian group actions, and the degree of symmetry of exotic spheres, Comment. Math. Helv. 49 (1974), 232-244.

16. J. Levine, Semifree circle actions on spheres, Invent. Math. 22 (1973), 161-186.

17. J. P. May, On kO-oriented bundle theories, Univ. of Chicago, 1974 (mimeographed).

18. J. Milnor, Remarks concerning spin manifolds, Differential and Combinatorial Topology (Sympos. in Homor of Marston Morse), Princeton Univ. Press, Princeton, N.J., 1965; pp. 55-62. MR 31 \#5208.

19. D. Quillen, The Adams conjecture, Topology 10 (1970), 67-80. MR 43 \#5525.

20. R. Schultz, Smooth structures on $s^{p} \times s^{q}$, Ann. of Math. (2) 90 (1969), 187198. MR $40 \# 3560$.

21. The nonexistence of free $S^{1}$ actions on some homotopy spheres, Proc. Amer. Math. Soc. 27 (1971), 595-597. MR 42 \#6866.

22. - Differentiable group actions on homotopy spheres. I, II (to appear).

23. J. Stasheff, The image of $J$ as a space mod $p$, Conf. on Algebraic Topology (Univ. of Illinois at Chicago Circle, Chicago, Ill., 1968), Univ. of Illinois at Chicago Circle, Chicago, III., 1969, pp. 276-287. MR 41 \#2679.

24. - (Editor), Problems proposed at the Conference, Univ. of Illinois at Chicago, Chicago, Ill., 1969, pp. 288-293.

25. C. T.C. Wall, Surgery on compact manifolds, London Math. Soc. Monographs, no. 1, Academic Press, New York, 1970.

DEPARTMENT OF MATHEMATICS, PURDUE UNIVERSITY, WEST LAFAYETTE, INDIANA 47907 